\title{
A CONJUGATION SYMMETRY IN LINEAR ELECTROMAGNETISM, IN EXTENSION OF MATERIALS WITH NEGATIVE REAL PERMITTIVITY AND PERMEABILITY SCALARS
}

\author{
Akhlesh Lakhtakia \\ CATMAS - Computational \& Theoretical Materials Sciences Group \\ Department of Engineering Science \& Mechanics \\ 212 Earth \& Engineering Sciences Building \\ Pennsylvania State University, University Park, PA 16802-6812
}

\begin{abstract}
If all space is occupied by a linear bianisotropic material — whether homogeneous or not - then the concurrent replacements of the permittivity and the impermeability tensors by the negatives of their respective complex conjugates and of the magnetoelectric tensors by their respective complex conjugates (in the Boys-Post representation) imply the conjugation of both $\underline{E}$ and $\underline{H}$, in the absence of externally impressed sources. This conjugation symmetry in linear electromagnetism has observable consequences when the linear bianisotropic material occupies a bounded region.
\end{abstract}

Keywords: bianisotropy; conjugate invariance; conjugation symmetry; negative permittivity; negative permeability; reflection; transmission

\section{INTRODUCTION}

The modest aim of this communication is to present a conjugation symmetry of frequencydomain electromagnetic fields in linear, nonhomogeneous, bianisotropic materials. This symmetry emerged as a generalization of a result obtained initially for linear, homogeneous, isotropic, dielectric-magnetic materials with negative real permittivity and permeability scalars [1]-[4]. Nominally, such a material possesses a relative permittivity scalar $\epsilon_{r}=\epsilon_{r}^{\prime}+i \epsilon_{r}^{\prime \prime}$ and a relative permeability scalar $\mu_{r}=\mu_{r}^{\prime}+i \mu_{r}^{\prime \prime}$, both dependent on the angular frequency $\omega$, such that both $\epsilon_{r}^{\prime}<0$ and $\mu_{r}^{\prime}<0$ in some spectral regime; accordingly, the phase velocity vector and the 
time-averaged Poynting vector are oppositely directed in that spectral regime [4]. ${ }^{1}$ Originally conceived more than 35 years ago [1], these materials were artificially realized quite recently [2].

During an investigation of changes in frequency-domain electromagnetic fields when the transformation $\left\{\epsilon_{r}^{\prime} \rightarrow-\epsilon_{r}^{\prime}, \quad \mu_{r}^{\prime} \rightarrow-\mu_{r}^{\prime}\right\}$ is effected on the isotropic dielectric-magnetic material occupying a source-free region, a more general conjugation symmetry in linear electromagnetism began to take shape. The following sections of this communication report the development of that symmetry.

\section{CONJUGATE INVARIANCE OF MAXWELL POSTULATES}

The frequency-domain Maxwell postulates may be written as

$$
\left.\begin{array}{l}
\nabla \cdot \underline{D}(\underline{r}, \omega)=\rho_{e}(\underline{r}, \omega) \\
\nabla \cdot \underline{B}(\underline{r}, \omega)=\rho_{m}(\underline{r}, \omega) \\
\nabla \times \underline{E}(\underline{r}, \omega)=i \omega \underline{B}(\underline{r}, \omega)-\underline{J}_{m}(\underline{r}, \omega) \\
\nabla \times \underline{H}(\underline{r}, \omega)=-i \omega \underline{D}(\underline{r}, \omega)+\underline{J}_{e}(\underline{r}, \omega)
\end{array}\right\},
$$

in the presence of externally impressed sources of the electric and magnetic types. These four postulates are collectively invariant with respect to the transformation

$$
\begin{aligned}
& \left\{\underline{E} \rightarrow \underline{E}^{*}, \underline{H} \rightarrow \underline{H}^{*}, \underline{D} \rightarrow-\underline{D}^{*}, \underline{B} \rightarrow-\underline{B}^{*},\right. \\
& \left.\quad \underline{J}_{e} \rightarrow \underline{J}_{e}^{*}, \underline{J}_{m} \rightarrow \underline{J}_{m}^{*}, \rho_{e} \rightarrow-\rho_{e}^{*}, \rho_{m} \rightarrow-\rho_{m}^{*}\right\},
\end{aligned}
$$

where the asterisk denotes the complex conjugate. This statement of conjugate invariance may be verified by direct substitution of (2) in (1).

The conjugate invariance of the Maxwell postulates not only underlies a similarly invariant Beltrami form of electromagnetism [6], but also permits the existence of a conjugation symmetry in linear electromagnetism.

\footnotetext{
${ }^{1}$ The condition for the phase velocity and the time-averaged Poynting vectors to be oppositely directed is $\left(\left|\epsilon_{r}\right|-\epsilon_{r}^{\prime}\right)\left(\left|\mu_{r}\right|-\mu_{r}^{\prime}\right)>\epsilon_{r}^{\prime \prime} \mu_{r}^{\prime \prime}$, which permits - more generally - $\epsilon_{r}^{\prime}$ and/or $\mu_{r}^{\prime}$ to be negative [5]. An $\exp (-i \omega t)$ time-dependence having been assumed here, $\epsilon_{r}^{\prime \prime}>0$ and $\mu_{r}^{\prime \prime}>0$ at all $\omega>0$ for all passive materials.
} 


\section{CONJUGATE INVARIANCE AND LINEAR BIANISOTROPY}

There are two widely used sets of frequency-domain constitutive relations for linear bianisotropic materials [7]. Both are considered as follows:

- Boys-Post constitutive relations. The Boys-Post constitutive relations of a linear, nonhomogeneous, bianisotropic material can be stated as

$$
\left.\begin{array}{l}
\underline{D}(\underline{r}, \omega)=\underline{\underline{\epsilon}}(\underline{r}, \omega) \cdot \underline{E}(\underline{r}, \omega)+\underline{\underline{\alpha}}(\underline{r}, \omega) \cdot \underline{B}(\underline{r}, \omega) \\
\underline{H}(\underline{r}, \omega)=\underline{\beta}(\underline{r}, \omega) \cdot \underline{E}(\underline{r}, \omega)+\underline{\underline{\chi}}(\underline{r}, \omega) \cdot \underline{B}(\underline{r}, \omega)
\end{array}\right\},
$$

subject to the constraint Trace $\{\underline{\underline{\alpha}}-\underline{\underline{\beta}}\} \equiv 0$. Whereas $\underline{\underline{\epsilon}}$ is the permittivity tensor and $\underline{\underline{\chi}}$ is the impermeability tensor, both $\underline{\underline{\alpha}}$ and $\underline{\underline{\beta}}$ are magnetoelectric tensors.

The transformation

$$
\left\{\underline{\underline{\epsilon}} \rightarrow-\underline{\underline{\epsilon}}^{*}, \underline{\underline{\chi}} \rightarrow-\underline{\underline{\chi}}^{*}, \underline{\underline{\alpha}} \rightarrow \underline{\underline{\alpha}}^{*}, \beta \rightarrow \underline{\underline{\beta}}^{*}\right\}
$$

of constitutive tensors then entails the transformation

$$
\left\{\underline{E} \rightarrow \underline{E}^{*}, \underline{H} \rightarrow \underline{H}^{*}, \underline{D} \rightarrow-\underline{D}^{*}, \underline{B} \rightarrow-\underline{B}^{*}\right\}
$$

of the electromagnetic fields - in conformity with (2) expressing the conjugate invariance of the Maxwell postulates.

- Tellegen constitutive relations. The Tellegen constitutive relations of a linear, nonhomogeneous, bianisotropic material can be stated as

$$
\left.\begin{array}{l}
\underline{D}(\underline{r}, \omega)=\underline{\hat{\epsilon}}(\underline{r}, \omega) \cdot \underline{E}(\underline{r}, \omega)+\underline{\underline{\xi}}(\underline{r}, \omega) \cdot \underline{H}(\underline{r}, \omega) \\
\underline{B}(\underline{r}, \omega)=\underline{\zeta}(\underline{r}, \omega) \cdot \underline{E}(\underline{r}, \omega)+\underline{\underline{\mu}}(\underline{r}, \omega) \cdot \underline{H}(\underline{r}, \omega)
\end{array}\right\},
$$

subject to the constraint Trace $\left\{\underline{\underline{\mu}}^{-1} \cdot(\underline{\underline{\xi}}+\underline{\underline{\zeta}})\right\} \equiv 0$. Here, $\underline{\underline{\hat{\epsilon}}}$ is the permittivity tensor, $\underline{\underline{\mu}}$ is the permeability tensor, and both $\underline{\underline{\xi}}$ and $\underline{\underline{\zeta}}$ are magnetoelectric tensors.

The transformation

$$
\left\{\underline{\underline{\hat{\hat{\epsilon}}}} \rightarrow-\underline{\underline{\hat{\epsilon}}}^{*}, \underline{\underline{\mu}} \rightarrow-\underline{\underline{\mu}}^{*}, \underline{\underline{\xi}} \rightarrow-\underline{\underline{\xi}}^{*}, \zeta \rightarrow-\underline{\underline{\zeta}}^{*}\right\}
$$


of constitutive tensors then also entails the field transformation (5) in conformity with the conjugate invariance of the Maxwell postulates.

Because the frequency-domain constitutive relations of the Boys-Post and the Tellegen types are inter-translatable, the constitutive-tensor transformations (4) and (7) are actually equivalent.

\section{CONJUGATION SYMMETRY IN LINEAR ELECTROMAGNETISM}

The deductions in Section 3 permit the enunciation of the following conjugation symmetry: If all space were to be occupied by a linear bianisotropic material — whether homogeneous or not - then a change of the constitutive tensors as per (4) would imply the conjugation of both $\underline{E}(\underline{r}, \omega)$ and $\underline{H}(\underline{r}, \omega)$, in the absence of externally impressed sources. If such sources are present, then the conjuagtion symmetry is expressed jointly by (2) and (4).

The effect of the transformation (4) would be observable even if the linear bianisotropic material were to be confined to a bounded region. For instance, imagine a slab of infinite transverse extent and uniform thickness, separating two vacuous half-spaces; the slab is made of a linear, isotropic, dielectric-magnetic material; and a linearly polarized, propagating, plane wave is incident on the slab. If the real parts of the permittivity and the permeability scalars of the slab were to change sign simultaneously, then the reflection and the transmission coefficients would have to be replaced by their respective complex conjugates [8]. The same conclusion holds if the slab were to be piecewise uniform in the thickness direction. The complex conjugation of reflection and transmission coefficients would essentially hold even if the slab were to be bianisotropic and plane-stratified, but a dependence on the polar angle of the incidence wavevector would also appear — as demonstrated elsewhere for chiral ferrosmectic slabs [9]. However, the conjugation of the reflection and the transmission coefficients would not hold on reversal of the signs of the real parts of the permittivity and permeability scalars of an isotropic, plane-stratified, dielectric-magnetic slab, if the incident plane wave were to evanescent.

The conclusions stated in the foregoing paragraph were obtained both by direct calculation and by application of the conjugation symmetry enunciated at the beginning of this section. The latter procedure is, of course, very simple; and it demonstrates the importance of the proffered 
symmetry in quickly determining the observable consequences of employing newly emerging materials (such as isotropic dielectric-magnetic materials with negative real permittivity and permeabilty scalars, and their anisotropic counterparts) for various applications.

\section{References}

[1] V.S. Veselago, The electrodynamics of substances with simultaneously negative values of $\epsilon$ and $\mu$, Sov Phys Usp 10 (1968) 509-514.

[2] R.A. Shelby, D.R. Smith and S. Schultz, Experimental verification of a negative index of refraction, Science 292 (2001) 77-79.

[3] C.G. Parazzoli, R.B. Greegor, K. Li, B.E.C. Koltenbah and M. Tanielian, Experimental verification and simulation of negative index of refraction using Snell's law, Phys Rev Lett $90(2003) 107401$.

[4] A. Lakhtakia, M.W. McCall and W.S. Weiglhofer, Brief overview of recent developments on negative phase-velocity mediums (alias left-handed materials), Arch Elektron Übertrag 56 (2002) 407-410.

[5] M.W. McCall, A. Lakhtakia and W.S. Weiglhofer, The negative index of refraction demystified, Eur J Phys 23 (2002) 353-359.

[6] A. Lakhtakia and W.S. Weiglhofer, Covariances and invariances of the Beltrami-Maxwell postulates, IEE Proc SMT 142 (1995) 262-266.

[7] W.S. Weiglhofer, A flavour of constitutive relations: The linear regime, In: S. Zouhdi, A. Sihvola and M. Arsalane (eds), Advances in electromagnetics of complex media and metamaterials, Kluwer, Dordrecht, The Netherlands, 2003.

[8] A. Lakhtakia, On planewave remittances and Goos-Hänchen shifts of planar slabs with negative real permittivity and permeability, Electromagnetics 23 (2003) 71-75.

[9] A. Lakhtakia, Handedness reversal of circular Bragg phenomenon due to negative real permittivity and permeability, Optics Express 11 (2003) 716-722. 